\title{
Evaluación in silico de una epidemia de influenza aviar AH5N1 con transmisión humano-humano: efecto de las medidas sanitarias en Valencia, Venezuela, 2012
}

\author{
Mariana Reggeti, Emilse Romero y Antonio Eblen-Zajjur
}

\section{In silico evaluation of an aviar influenza AH5N1 virus outbreak with human to human transmission: effects of sanitary measures in Valencia, Venezuela, 2012}

Background: There is a risk for an avian influenza AH5N1 virus pandemia. Aim: To estimate the magnitude and impact of an AH5N1 pandemic in areas of Latin-America in order to design interventions and to reduce morbiditymortality. Methods: The InfluSim program was used to simulate a highly pathogenic AH5N1 aviar virus epidemic outbreak with human to human transmission in Valencia, Venezuela. We estimated the day of maximal number of cases, number of moderately and severely ill patients, exposed individuals, deaths and associated costs for 5 different interventions: absence of any intervention; implementation of antiviral treatment; reduction of $20 \%$ in population general contacts; closure of $20 \%$ of educational institutions; and reduction of $50 \%$ in massive public gatherings. Simulation parameters used were: population: 829.856 persons, infection risk 6-47\%, contagiousness Index $\mathrm{Rh}_{\mathrm{o}} 2,5$; relative contagiousness $90 \%$, overall lethality $64,1 \%$ and, costs according to the official basic budget. Results: For an outbreak lasting 200 days direct and indirect deaths by intervention strategies would be: 29,907; 29,$900 ; 9,701 ; 29,295$ and 14,752. Costs would follow a similar trend. Discussion: Reduction of $20 \%$ in general population contacts results in a significant reduction of up to $68 \%$ of cases. The outbreak would collapse the health care system. Antiviral treatment would not be efficient during the outbreak. Interpersonal contact reduction proved to be the best sanitary measure to control an AH5N1 theoretical epidemic outbreak.

Key words: Influenza; outbreak; prevention; AH5N1; simulation, social distance.

Palabras clave: Influenza; AH5N1; epidemia; prevención; simulación, distanciamiento social.

\section{Introducción}

os brotes de influenza aviar por la cepa AH5N1 reportados recientemente en diferentes partes del mundo, en especial en Asia, constituyen un recordatorio estricto de la amenaza de una pandemia, la cual causaría una enorme morbi-mortalidad y costos económicos considerables, especialmente en las zonas más desasistidas de los países expuestos debido a los factores de riesgo como estados de salud y nutrición deficientes así como los limitados recursos para vigilancia y asistencia de salud disponibles en ellos ${ }^{1}$.

En la actualidad, la influenza aviar AH5N1, constituye una de las mayores preocupaciones en salud pública dado su considerable potencial pandémico ${ }^{2}$. Las pandemias de influenza ocurren en ciclos de 20 a 30 años. La gran pandemia de influenza "Española" de 1918-19, (cepa AH1N1) causó 40 a 50 millones de muertes en todo el mundo. En 1957 ocurrió la influenza "Asiática”, (cepa AH2N2), en 1968 la influenza "Hong Kong”, (cepa AH3N2) y en 1977 la influenza "Rusa", (cepa AH1N1)³. En las más recientes se usaron vacunas, la intervención considerada más importante para la época en reducir la morbilidad y la mortalidad, pero éstas se implementaron tardíamente sin los beneficios esperados, generando dudas sobre su uso durante las epidemias y planteando la potencialidad de otras medidas sanitarias como el aislamiento individual (distanciamiento social) y de grupos de alto riesgo ${ }^{1,4}$.

Actualmente el riesgo de una pandemia es alto dado el reporte de casos animales y humanos en diferentes áreas. Los casos humanos ofrecen la oportunidad para una mutación viral y su transformación en una cepa pandémica ${ }^{1}$. En 1997, la cepa aviar AH5N1 apareció en Hong Kong con casos y mortalidad humana. Reapareció en 2003 en aves de corral en Vietnam, Indonesia y Tailandia con potencial extensión al resto de Asia, Europa y África ${ }^{5}$. En mayo de 2005, el virus AH5N1 mató más de 6.000 aves migratorias en China occidental ${ }^{5}$.

La epidemia de sindrome respiratorio agudo grave (SARS, del inglés) del 2002-2003 es un buen punto de referencia en costos y consecuencias sociales de las pandemias virales respiratorias ya que alcanzó los $\$ 50.000$ millones en pérdidas en el mundo, causando problemas sociales y alarma pública considerables, incluso en zonas y países donde no se produjeron casos. Se cerraron hospitales, colegios y algunas fronteras. Miles de per-
Universidad de Carabobo. Facultad de Ciencias de la Salud, Centro de Biofísica y Neurociencia, CBN-UC El Trigal, Valencia, Venezuela.

Los autores declaran la inexistencia de conflictos de interés.

Financiamiento: Centro de Biofísica y Neurociencia, Facultad de Ciencias de la Salud, Universidad de Carabobo. Valencia, Venezuela.

Recibido: 8 de septiembre de 2015 Aceptado: 20 de marzo de 2016

Correspondencia a: Antonio Eblen-Zajjur aeblen@uc.edu.ve 
sonas se pusieron en cuarentena de forma voluntaria o impuesta. El impedimento de viaje a determinadas zonas y el uso generalizado de mascarillas quirúrgicas fueron desproporcionados en comparación con el riesgo. Tanto los pacientes como los grupos étnicos se enfrentaron a la discriminación ${ }^{3}$.

Los virus de influenza pertenecen a la familia Orthomyxoviridae que comprende virus ARN. Su denominación surge de las proteínas hemaglutinina $(\mathrm{H})$ y neuraminidasa $(\mathrm{N})$ presentes en la superficie viral ${ }^{5}$. Mutaciones en una o ambas proteínas hace que ninguna persona posee inmunidad pudiendo surgir una pandemia cuando simultáneamente el virus adquiere la capacidad de transmitirse de persona a persona ${ }^{3}$.

Las aves acuáticas salvajes son el reservorio y portador asintomático de todos los subtipos de virus de influenza $\mathrm{A}^{5-9}$, con la excepción del subtipo H1N33 ${ }^{5,6}$. La infección de estas aves con el virus AH5N1 ha sido limitada y ocasional $^{10}$ variando su mortalidad ${ }^{11}$. El virus AH5N1 ha sido aislado de cerdos ${ }^{12}$, sugiriendo el paso de patos a cerdos por cohabitación, lo cual es preocupante, ya que los cerdos actúan como recipientes de recombinación de virus de la influenza humana y aviar ${ }^{13}$. Adicionalmente, el virus sobrevive en carnes de aves crudas y puede transmitirse a través de la carne congelada ${ }^{14}$ además de encontrarse dentro y fuera de los huevos.

La infección con AH5N1 en humanos es poco frecuente y se asocia al contacto directo o cercano con aves de corral infectadas. Produce fiebre, tos, dificultad respiratoria aguda, disnea, dolor abdominal y diarrea. Las complicaciones más frecuentes son neumonía, insuficiencia respiratoria, insuficiencia metabólica, alteración mental, convulsiones, fallo multiorgánico y muerte ${ }^{15}$. La mortalidad humana reportada alcanza hasta $64,1 \%{ }^{16}$, con alta incidencia de neumonía $(61 \%)$ y de casos con un requerimiento de cuidados intensivos de hasta $51 \%{ }^{4}$.

Venezuela es una comprobada área de migración y permanencia de cerca de 153 especies de aves acuáticas en estrecha relación con las actividades de poblaciones humanas. Cerca de $60 \%$ de las especies son consideradas de alto riesgo y casi $60 \%$ son migratorias. El Estado Carabobo y el Lago de Valencia constituyen una de las áreas con más especies de aves ${ }^{17}$. La Red Mundial de Vigilancia Epidemiológica de Influenza Aviar en Aves Silvestres (GAINS, siglas en inglés) ya ha detectado 25 especies de aves acuáticas propensas a contraer y a dispersar el virus AH5N1 en las costas y humedales venezolanos ${ }^{18}$. La ciudad de Valencia, capital estatal, se ubica en las cercanías del lago epónimo, posee condiciones propicias para que las aves con potencial diseminador del virus AH5N1 entren en contacto con otros animales de corral y con la población humana, condiciones propicias para el inicio de la epidemia aviar, lo que unido a la potencial transmisión humano-humano, es el objetivo del presente estudio.
Para reducir al mínimo la repercusión de una pandemia, es preciso elaborar con anticipación la planificación y ejecución de actividades que permitan identificar los efectos, daños y comportamiento poblacional, así como las posibles estrategias preventivas, medidas sanitarias, tratamientos específicos y sus potenciales efectos. Una forma de cumplir estos objetivos es el uso de herramientas de simulación epidemiológica que aplicando modelos matemáticos poblacionales permiten simular escenarios de desastres ${ }^{19} \mathrm{o}$ epidemias ${ }^{20} \mathrm{y}$ de intervención en ellos aportando datos relevantes para la planificación de estrategias tendientes a reducir su impacto; tal es el caso de simuladores específicos de epidemias de influenza como InfluSim, QuickFlu y EpiDyNet ${ }^{21-23}$.

En el presente estudio se evaluó in silico el impacto de una epidemia del virus de la influenza aviar altamente patógena $\mathrm{AH} 5 \mathrm{~N} 1$ sobre la población de la ciudad de Valencia, Estado Carabobo, Venezuela, para el año 2012, en un escenario de carácter pandémico, es decir, asumiendo una transmisión humano a humano con un número de reproducción básico reportado $\left(\mathrm{H}_{\mathrm{o}}=2,5\right)$ para casos humanos y de los resultados de las medidas sanitarias conocidas para tal situación.

\section{Materiales y Métodos}

Se trata de una investigación descriptiva, experimental, transeccional, in silico.

\section{Características geográficas de la ciudad de Valencia}

La ciudad de Valencia, se ubica geográficamente en $68^{\circ} 07^{\prime}$ y $67^{\circ} 48^{\prime} \mathrm{O}$ y $10^{\circ} 14^{\prime}$ y $9^{\circ} 49^{\prime} \mathrm{N}$, cuenta con $623 \mathrm{~km}^{2}$, su temperatura media anual es de $26,7^{\circ} \mathrm{C}$, la precipitación promedio anual es de 1.138,2 mm con el período lluvioso de mayo a noviembre y un máximo en el mes de agosto $^{19,24}$.

\section{Programa de simulación}

InfluSim v1.1 es un modelo determinístico compartamental basado en un sistema de 1.081 ecuaciones diferenciales creado por Eichner y cols., (2007) ${ }^{21-23}$. El simulador extiende el modelo dinámico SEIR (Susceptibles-Expuestos-Infectados-Recuperados) clásico con los parámetros clínicos y demográficos relevantes para la planificación de intervenciones para una pandemia ${ }^{21-23}$ así como los postulados de modelaje estático ${ }^{25}$. Permite generar cursos temporales y valores acumulativos de casos de influenza, visitas ambulatorias, dosis aplicadas de tratamiento antiviral, hospitalizaciones, muertes y días de trabajo perdidos debido a la enfermedad y sus $\operatorname{costos}^{21-23,25}$. Reproduce la dinámica de infección de la gripe pandémica ofreciendo gran reproducibilidad, por cuanto se basa en un modelo determinístico, con alto 


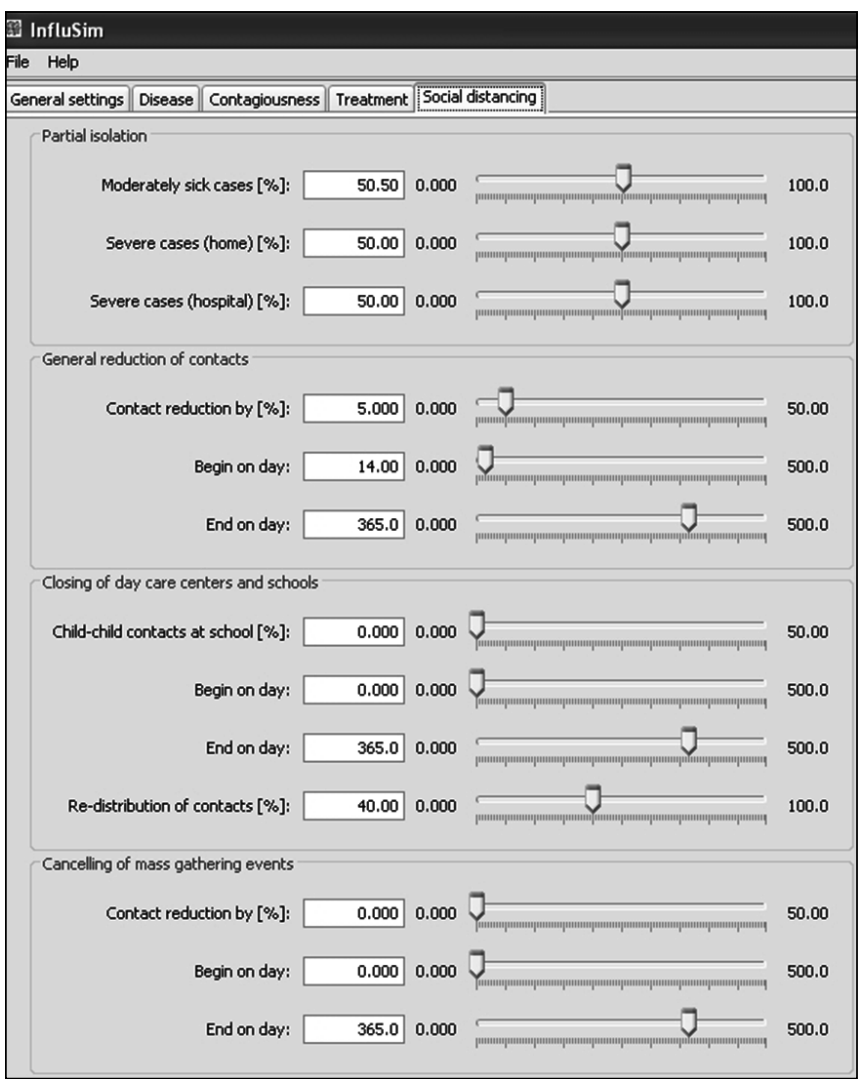

Figura 1. Interfase del usuario de InfluSim v1.1. Panel para definiciones de los parámetros del modelo generales, enfermedad, contagiosidad, tratamiento y distanciamiento social, este último desplegado para definir el aislamiento parcial, reducción general de contactos así como el día de cierre de los colegios y eventos de asistencia masiva.

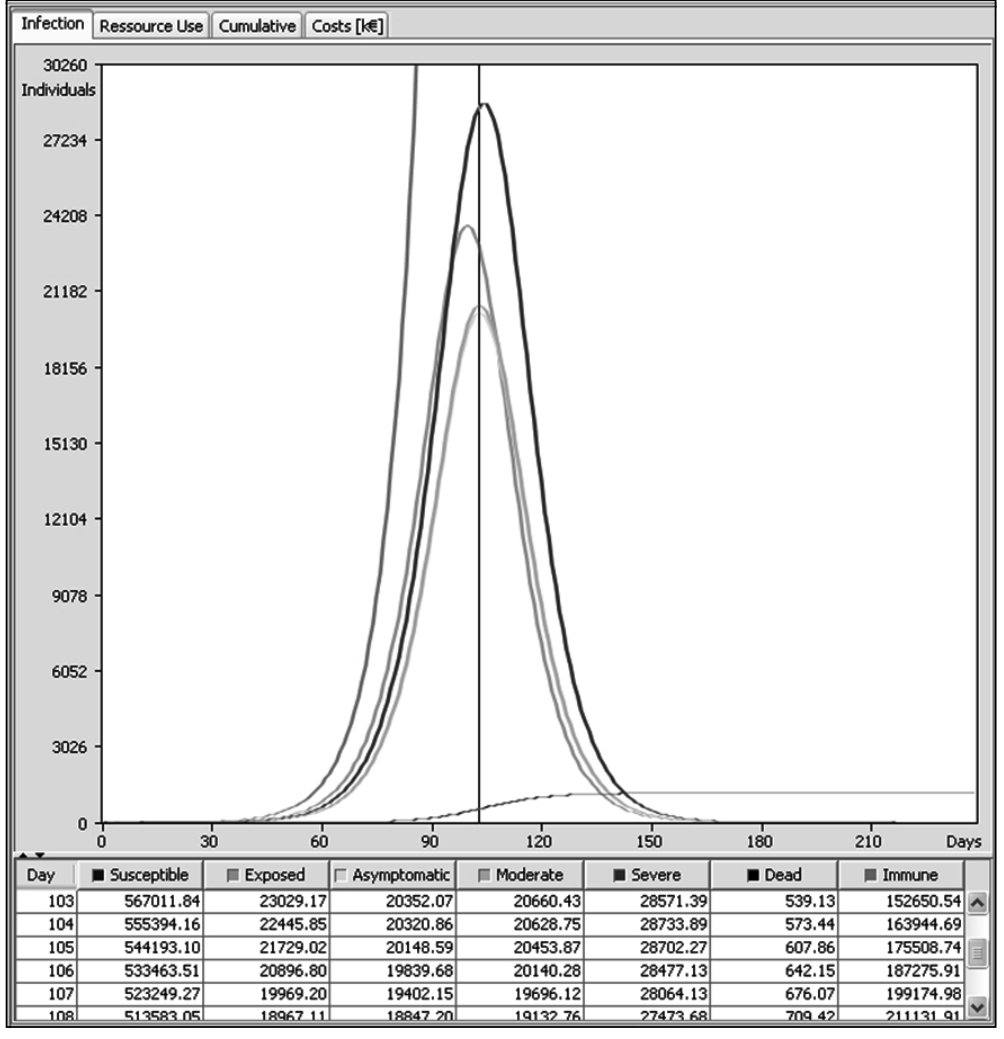

Figura 2. Interfase del usuario de InfluSim v1.1. Panel de resultados gráficos sobre infección, recursos, valores acumulados y costos. Se desplegó el panel gráfico correspondiente al curso temporal de la epidemia en días (panel superior) con las curvas de las cantidades diarias de casos susceptibles, expuestos, asintomáticos, enfermos moderados y severos, muertes e inmunes cuyos valores numéricos diarios aparecen listados en el panel inferior. El cursor vertical indica los valores del día 104 de la epidemia, las curvas se diferencian por colores. rendimiento computacional a pesar del complejo sistema de ecuaciones diferenciales que utiliza para obtener las soluciones y operatividad ${ }^{21-23}$. La interfase del usuario del simulador InfluSim se presenta en las Figuras 1 y 2.

El modelo se ajustó a las características poblacionales de la ciudad de Valencia y de la cepa de AH5N1 para evaluar el curso temporal del número de individuos susceptibles, expuestos, asintomáticos, enfermos moderados (hospitalizados sin soporte ventilatorio), graves (con soporte ventilatorio), muertos e inmunes, el costo económico por días laborables perdidos, consultas, hospitalizaciones, tratamiento antiviral y totales, así como momento máximo y duración de una epidemia en cinco escenarios: sin intervención sanitaria (SI); tratamiento antiviral masivo (AV); aislamiento social en $20 \%$ (AS); cierre de $20 \%$ de los colegios (CC) y reducción de $50 \%$ de los eventos de reunión masiva de personas. Finalmente, se contrastó los requerimientos calculados para la atención pública durante la epidemia con la capacidad instalada de atención sanitaria en la ciudad para el año 2012, año del que se dispone de cifras epidemiológicas completas ${ }^{26}$.

\section{Parámetros de simulación}

Población de Valencia: Población menor (menores de 15 años): 206.351 (24,87\%), Población económicamente productiva (15-64 años): 571.785 (68,09\%), Población mayor (mayores de 64 años): $51.720(6,23 \%)$ para un total de 829.856 habitantes $^{26}$.

Porcentaje de riesgo: Población menor (menores de 15 años): $6 \%$, Población económicamente productiva (15-64 años): 14\%, Población mayor (mayores de 64 años): $47 \%{ }^{21-23,26-28}$

Contagiosidad: Medido por el coeficiente $\mathrm{R}_{0}$ (con valor para cepas pandémicas entre 2 y 3 ). El valor usado fue 2,5 y una contagiosidad relativa de $90 \% \%^{21-23,25,27,29-32}$.

Costos (año 2012): Canasta Básica Familiar BsF. 26.576,00 (US\$ 200,00); Salario Mínimo BsF. 4.251,00 (US\$ 85,02); Canasta Básica Familiar Semanal BsF. 6.644 (US\$ 132,88); Costo de Antivirales (Oseltamivir) BsF. 5.000,00 (US\$ 100,00) por 10 días de tratamiento $^{21-23,25,26,33-38}$.

Enfermedad: Duraciones promedios, gravedad de la enfermedad, fracción de casos hospitalizados/casos gra- 
ves, tasa de letalidad global: $64,1 \%$; $56 \%$ para niños, $42 \%$ para pacientes trabajadores y $64 \%$ para los ancianos ${ }^{16,21-23}$.

\section{Análisis estadístico}

Los resultados de los escenarios de simulación se presentan como tablas. Los valores porcentuales de los diferentes escenarios se compararon mediante el cálculo del valor $z^{39}$ y la prueba de $\chi^{2}$ para las tablas de contingencia, considerándose como significativos valores de $z \geq 1,96(\mathrm{p}<0,05)$.

\begin{tabular}{|c|c|c|c|c|c|}
\hline Variable & SI & AV $(\Delta \%)$ & AG $(\Delta \%)$ & Coleg $(\Delta \%)$ & $\operatorname{EM}(\Delta \%)$ \\
\hline Día máximo & 104 & $104 \quad(0)$ & $165(+58,7)$ & $108(+3,8)$ & $133(+27,9)$ \\
\hline Moderados & 20.660 & $20.653(-0,03)$ & $6.517(-68,5)$ & $19.682(-4,7)$ & $10.547(-48,9)$ \\
\hline Graves & 28.734 & $28.728(-0,02)$ & $9.035(-68,6)$ & $28.071 \quad(-2,3)$ & $14.048(-51,1)$ \\
\hline Muertes & 1.173 & $1.172(-0,08)$ & $666(-56,7)$ & $1.224(+4,3)$ & $706(-60,1)$ \\
\hline
\end{tabular}

Sombreado: $p<0,0001$ comparado con escenario sin intervención sanitaria (SI). $\chi^{2}=1161,7$; $p<0,0001$ tabla de cantidad de casos e intervenciones. *Escenarios teóricos: SI: sin intervención sanitaria; AV: tratamiento antiviral; AG: reducción en $20 \%$ de los contactos generales; Coleg: cierre de $20 \%$ de los colegios; EM: prohibición de reuniones masivas en $50 \%$.

\begin{tabular}{|c|c|c|c|c|c|}
\hline Variable & SI & AV $(\Delta \%)$ & AG $(\Delta \%)$ & Coleg $(\Delta \%)$ & $\operatorname{EM}(\Delta \%)$ \\
\hline Semanas perdidas & 160.697 & $160.593(-0,06)$ & $93.873(-41,6)$ & $168.476(+4,8)$ & $95.820(-40,4)$ \\
\hline Consultas & 111.641 & $111.582(-0,05)$ & $66.744(-40,2)$ & $112.520(+0,8)$ & $74.988(-32,8)$ \\
\hline Hospitalización & 1.698 & $1.697(-0,06)$ & $989(-58,1)$ & $1.774(+4,5)$ & $1.021(-39,9)$ \\
\hline
\end{tabular}

Tabla 3. Costo en dólares por días laborales perdidos, consultas médicas o por hospitalización en epidemia de influenza aviar subtipo AH5N1 en Valencia, Venezuela, 2010, en cinco escenarios teóricos*, expresados como valores absolutos y porcentaje de cambio en relación a los valores de SI

\begin{tabular}{|c|c|c|c|c|c|}
\hline Variable & SI & $\operatorname{AV}(\Delta \%)$ & AG $(\Delta \%)$ & Coleg $(\Delta \%)$ & EM $(\Delta \%)$ \\
\hline Días perdidos & 157.209 & $157.134(-0,05)$ & $91.902(-41,5)$ & $164.930(+4,9)$ & $93.716(-40,4$ \\
\hline Consultas & 168.031 & $167.948(-0,05)$ & $100.489(-40,2)$ & $169.453(+0,8)$ & $112.861(-32$ \\
\hline Hospitalización & 178.898 & $178.972(-0,06)$ & $104.199(-41,8)$ & $186.905(+4,5)$ & 107.570 \\
\hline osto tota & 504.139 & $03.875(-0,05)$ & $296.590(-41,2)$ & $521.260(+3,4)$ & $314.148(-37$ \\
\hline
\end{tabular}

Sombreado: $p<0,0001$ comparado con escenario sin intervención sanitaria $(\mathrm{SI}) \cdot \chi^{2}=1,04$; p > 0,99 Tabla $3 \times 5$. *Ver pie de Tabla 1.

\section{Resulltados}

La Tabla 1 presenta los valores comparativos de los cinco escenarios epidémicos evaluados en torno al número de casos de la enfermedad. Al realizar las comparaciones se evidenció que no existen diferencias estadísticamente significativas entre la ausencia de intervención sanitaria y los resultados obtenidos por medidas como el tratamiento antiviral o el cierre de $20 \%$ de los contactos en colegios. Sin embargo, la reducción de $20 \%$ en los contactos en la población general sí produjo una reducción significativa de hasta $68 \%$ en los números de casos, así como en el retardo del pico máximo de la epidemia en $58 \%$ para la reducción de $20 \%$ de los contactos en la población general a $27 \%$ en el caso de la reducción de $50 \%$ en los eventos multitudinarios.

Resultados similares en estas intervenciones se aprecian al evaluar las semanas laborales perdidas, en consulta médica o en hospitalización en los escenarios de epidemia (Tabla 2), siendo las intervenciones más efectivas nuevamente la reducción de $20 \%$ de los contactos en la población general y la reducción del $50 \%$ en los eventos multitudinarios. De igual forma, al analizar las pérdidas monetarias generadas por cada escenario epidémico, nuevamente la reducción de $20 \%$ de los contactos en la población general y la reducción de $50 \%$ en los eventos multitudinarios reducen significativamente los costos durante el tiempo de duración de la epidemia (Tabla 3).

\section{Discusión}

En el presente estudio se utilizó el Programa de simulación InfluSim versión 1.1 para modelar una epidemia de virus influenza aviar altamente patógeno, subtipo AH5N1, en la población de la Ciudad de Valencia, Estado Carabobo, Venezuela. Usando los parámetros demográficos del Censo Oficial del año 2012, se generaron por primera vez, a nuestro entender, estimados del día de máximo número de casos, enfermos moderados, graves, expuestos y muertes, así como de los costos materiales de dicha epidemia en los cinco escenarios propuestos: sin intervención sanitaria; tratamiento antiviral; reducción de $20 \%$ del contacto general en la población; cerrando $20 \%$ de los colegios e instituciones educativas y reduciendo en $50 \%$ las reuniones públicas masivas.

Los resultados arrojan cifras devastadoras para la población en todos los escenarios. En el caso de ausencia de medidas sanitarias, se generaron más de 28.000 casos graves con requerimiento de hospitalización y cuidados urgentes especiales, la gran mayoría de éstos con ventilación asistida. En este modelo, el resultado de muertes directas es de 1.173 casos para aproximadamente 200 días de epidemia; sin embargo, el desproporcionado número de casos graves que no podrían recibir atención crítica 
respiratoria adecuada por el colapso de todo el sistema de salud, no sólo regional sino nacional, produciría un número considerablemente mayor de defunciones a expensas de las cifras de casos graves (4), pudiendo llegar conservadoramente a $50 \%$ de los casos graves lo que representa cerca de 14.000 muertes. La disponibilidad de camas para cuidados intensivos en la ciudad y el Estado Carabobo de la cual es su capital, no sobrepasa de 90 incluyendo la red de instituciones públicas y privadas, a lo que habría que añadir el impacto directo de la enfermedad en el grupo de más alto riesgo que corresponde al del propio equipo de salud, el cual se vería considerablemente reducido por la misma enfermedad y/o deserción, fenómeno ya descrito en otras epidemias $23,25,27,29,31,32$.

Al evaluar por separado las estrategias de intervención sanitaria, la más eficiente fue la reducción en los contactos generales en la población. En el presente estudio se probó una reducción de $20 \%$ en estos contactos poblacionales lo cual redujo en $56 \%$ el número de muertes y en más de $68 \%$ los casos graves y moderados retardando en $58 \%$ el pico epidémico. Esta medida conocida como distanciamiento social ${ }^{22,29,30,32,37,38}$, se pone en práctica solicitando a la población permanecer en sus hogares y reducir a lo estrictamente necesario los contactos con otras personas. Una medida similar como es la restricción de reuniones públicas aporta resultados parecidos pero con aproximadamente $10 \%$ menor en su eficiencia. Es probable que la combinación aporte un efecto sinérgico ${ }^{22,23,25,27}$. Estos resultados concuerdan parcialmente con reportes previos $^{22,23,25,27,29}$ en los que evaluaciones in silico de epidemias de influenza han resultado en mejores controles mediante la combinación del distanciamiento social con el tratamiento antiviral, con un índice de reproducción viral $\mathrm{R}_{0}<1,8$ y con una cobertura considerable del tratamiento antiviral iniciado muy tempranamente. Estas condiciones difícilmente podrían darse en la población de estudio ya que el $\mathrm{R}_{0}$ usado fue de 2,5 basado en los promedios reportados en epidemias previas ${ }^{21-23,25,27,29-32}$ y se suma a la falta de disponibilidad inmediata del antiviral.

Desde el punto de vista del número de consultas y de hospitalizaciones, así como de sus costos, las cifras son desalentadoras en todos los escenarios, con un impacto negativo considerable en la economía familiar, regional y nacional.

El tratamiento con medicación antiviral no mostró ser eficiente en alguno de los escenarios en los que se incluyó, lo cual contrasta con la efectividad reportada del inhibidor de la neuramidinasa incluido en el modelo (oseltamivir). Una explicación de estos resultados son las condiciones aditivas para el uso eficiente del antiviral durante la epidemia, entre las que se encuentran la necesidad de iniciar el tratamiento en menos de tres días después de la infección, lograr la mayor cobertura en la fuente de la epidemia, disponibilidad suficiente de la medicación para todos los grupos de alto riesgo y sospecha de contactos y ausencia de resistencia ${ }^{31,32,38,40}$.

Los resultados de la presente investigación sugieren fuertemente la necesidad de preparar y educar a la población en las estrategias de reducción de contactos y reducción del riesgo de infección que constituyen medidas de bajo costo. Aditivamente se requiere mantener la vigilancia ambiental en la detección temprana de mortalidad en aves migratorias, reevaluar el uso de los medicamentos antivirales y otras estrategias como la vacunación y su disponibilidad poblacional. Finalmente, se requiere incrementar la capacidad de atención del sistema de salud con planes de contingencia ensayados para reducir el impacto directo en los miembros del equipo de salud y en la población ante una situación de magnitud extrema.

\section{Resumen}

Introducción: Existe el riesgo de una pandemia de influenza aviar por virus AH5N1. Objetivo: Conocer la magnitud e impacto en áreas latinoamericanas de una pandemia AH5N1 a fin de planificar las medidas sanitarias para reducir la morbimortalidad. Material y Método: Mediante el simulador InfluSim se modeló una epidemia por virus AH5N1 con transmisión Humano-Humano, en Valencia, Venezuela. Se calculó el día de máximo número de casos, cantidad de enfermos moderados, graves, expuestos, muertos, y costos en cinco escenarios diferentes: sin intervención sanitaria; tratamiento antiviral; reducción en $20 \%$ del contacto en la población; cierre de $20 \%$ de las instituciones educativas; reducción de $50 \%$ de las reuniones públicas. Parámetros usados: Población: 829.856 habitantes, Porcentaje de riesgo 6-47\%, Contagiosidad índice $\left(R_{0}\right)$ 2,5; Contagiosidad relativa 90\%, Tasa de letalidad global 64,1\%, costos según Canasta Básica oficial. Resultados: En 200 días de epidemia: Muertes totales por escenario: a: 29.907; b: 29.900; c: 9.701; d: 29.295 y d: 14.752. Similar tendencia en costos. Discusión: Reducir $20 \%$ los contactos de la población produjo una reducción significativa de $68 \%$ en el número de casos. La epidemia colapsará los sistemas de salud disponibles por cantidad de casos. El tratamiento antiviral no es eficiente durante la epidemia. La reducción en los contactos interpersonales muestra ser la mejor medida sanitaria.

\section{Referencias bibliográficas}

1.- World Health Organization (WHO). WHO/ CDS/CSR/GIP/2005.8. Respuesta a la amenaza de una pandemia de gripe aviar. Medidas estratégicas recomendadas. Enfermedades Transmisibles (Vigilancia y Respuesta). Programa Mundial de la Gripe [online]. Disponible en: http://www.who.int/influenza/ resources/documents/h5n1_strategic_actions/es/
(Accedido el 17 de febrero de 2015).

2.- Red Internacional de Autoridades de Inocuidad de los Alimentos (INFOSAN). Nota de información INFOSAN No ${ }^{\circ}$ /04-Gripe aviar, de 17 de diciembre de 2004. [online]. Disponible 
en: http://www.who.int/foodsafety/fs management/No_07_AI_Nov05_sp.pdf?ua $=1$. (Accedido el 28 de septiembre de 2014).

3.- Organización Mundial de la Salud. WHO/CDS/ CSR/GIP/2004. Reunión de consulta sobre los preparativos para una pandemia de influenza en países con recursos limitados. Kuala Lumpur, Malasia, 23-25 de junio de 2004; 1: 1,2.

4.- Arrasco J C, Gómez J L. Guía de investigación de brotes de infecciones respiratorias agudas e influenza, Lima 2007. Ministerio de Salud; Dirección General de Epidemiología. pp 60.

5.- Wright P F, Neumann G, Kawaoka Y. Orthomyxoviruses. En: DM Knipe, Howley PM, Griffin DE, et al. eds. Fields in Virology. Philadelphia: Wolters Kluwer, Lippincott Williams \& Wilkins; 2007: 1691740.

6.- Ellis T M, Bousfield R B, Bissett L A, Dyrting $\mathrm{K} \mathrm{C}$, Luk G S, Tsim S T, et al. Investigation of outbreaks of highly pathogenic $\mathrm{H} 5 \mathrm{~N} 1$ avian influenza in waterfowl and wild birds in Hong Kong in late 2002. Avian Pathol 2004; 33: 492505.

7.- Maines T R, Lu X H, Erb S M, Edwards L, Guarner J, Greer P. Avian influenza (H5N1) viruses isolated from humans in Asia in 2004 exhibit increased virulence in mammals. $\mathrm{J}$ Virol 2005; 79 (18), 11788-11800. doi:10.1128/ JVI.79.18.11788-11800.2005.

8.- Chen H, Smith G J, Li K S. Establecimiento de múltiples sublinages de virus de la gripe $\mathrm{H} 5 \mathrm{~N} 1$ en Asia: implicaciones para el control de la pandemia. Proc Natl Acad Sci EE. UU. 2006.

9.- Wang J, Vijaykrishna D, Duan L, Bahl J, Zhang $\mathrm{J} X$, Webster R G, et al. Identification of the progenitors of Indonesian and Vietnamese avian influenza $\mathrm{A}(\mathrm{H} 5 \mathrm{~N} 1)$ viruses from southern China. J Virol 2008; 82 (7): 3405-14.

10.- Becker W B. The isolation and classification of often virus: Influenza virus A-tern/South Africa/1961. J Hyg 1966; 64: 309-20.

11.- Chen H, Li Y, Li Z, Shi J, Shinya K, Deng G, et al. Properties and dissemination of H5N1 viruses isolated during an influenza outbreak in migratory waterfowl in western China. J Virol 2006; 80: 5976-83.

12.- Chen H, Deng G, Li Z, Tian G, Li Y, Jiao P, et al. The evolution of H5N1 influenza viruses in ducks in southern China. Proc Natl Acad Sci USA. 2004; 101: 10452-7.

13.- Scholtissek C, Burger H, Kistner O, Shortridge $\mathrm{K}$ F. La nucleoproteína como un posible factor importante en la determinación de la especificidad del hospedador del virus H3N2 de la gripe. Virología 1985; 147: 287-94.

14.- Brotes en aves de corral y seres humanos de influenza aviar altamente patógena $\mathrm{H} 5 \mathrm{~N} 1$ : las implicaciones en inocuidad de los alimentos. INFOSAN nota informativa $\mathrm{N}^{\circ}$ 2/04-influenza aviar; 17 de diciembre de 2004

15.- Documento $3^{\circ}$ del Depto. de Salud y Servicios
Humanos de EE. UU. 200 Independence Avenue, S.W. - Washington, D.C. 2020.

16.- Organisation Mondiale de la Santé Genève. Weekly epidemiological record. Relevé épidémiologique hebdomadaire. 11 JULY 2014, 89th year/11 No 28, 2014, 89, 309-320. [http:// www.who.int/wer/2014/wer8928.pdf?ua=1].

17.- Martínez M, Giner S. Venezuela: Informe Anual Censo Neotropical de Aves Acuáticas 2010. Wetlands Internacional. [online]. Disponible en: http://lac.wetlands.org/Nuestrasactividades/ Conservaci\%C3\%B3ndeAvesAcu\%C3\%A1tic as/Censoneotropicaldeavesaqu $\% \mathrm{C} 3 \% \mathrm{~A} 1$ ticas/ Comoparticipar/Venezuela/tabid/1246/language/ es-ES/Default.aspx. (Accedido el 10 de agosto de 2014).

18.- Wetlands Internacional. Mapeo de distribución, abundancia y rutas migratorias de aves acuáticas en América del Sur. Donante WCS/GAINS program/USAID. [online]. Disponible en: http://lac.wetlands.org/Loquehacemos/Cons ervaci $\% \mathrm{C} 3 \% \mathrm{~B} 3$ ndeAvesAcu $\% \mathrm{C} 3 \% \mathrm{~A} 1$ ticas/ Mapasdeavesacu\%C3\%A1ticas/tabid/1624/ Default.aspx. (Accedido el 10 de agosto de 2014).

19.- Bosco R, Malpica O, Eblen-Zajjur A. Simulación de una explosión nuclear en Valencia, Venezuela: Análisis biofísico y médico. Interciencia 2004; 29: 485-9.

20.- Samudio M, Vesga J F, Cohenca B, Jacobs M, Brezzo C. Estimación de la incidencia de VIH en población adulta de Paraguay con el modelo matemático MoT. Rev Panam Salud Pública 2015; 37 (3): 154-61.

21.- Eichner M, Schwehm M, Duerr H-P, Brockmann S O. The influenza pandemic preparedness planning tool InfluSim. BMC Infect Dis 2007; 7: 17-25.

22.- Duerr H P, Stefan O, Brockmann S O, Piechotowski I, Schwehm M, Eichner M. Influenza pandemic intervention planning using InfluSim: pharmaceutical and nonpharmaceutical interventions. BMC Infect Dis 2007; 7: 76-89.

23.- Chu Ch, Lee J, Choi D H, Youn S-K, Lee J-K. Sensitivity analysis of the parameters of Korea's pandemic influenza preparedness plan. Osong Pub Health Res Persp 2011; 2: 210-5.

24.- Instituto Geográfico de Venezuela Simón Bolívar (IGVSB). Disponible en: http://sia. geoportalsb.gob.ve/sia/ (accedido el 15 de agosto de 2014).

25.- Meltzer M I, Cox N J, Fukuda K. The economic impact of pandemic influenza in the United States: priorities for intervention. Emerg Infect Dis 1999; 5: 659-71.

26.- Instituto Nacional de Estadística (INE), Censo 2011. Gerencia de Estadísticas Ambientales. Informe Geoambiental 2011. Estado Carabobo. Municipio Valencia. 162-72.

27.- Anonymous: Influenza pandemie planung: Nationaler Influenza pandemie plan.
Bundesgesundheitsblatt - Gesundheitsforschung - Gesundheitsschutz 2005; 48: 356-90.

28.- Instituto Nacional de Estadística (INE), Censo 2011. Procesado con Redatam + SP, CEPAL/ CELADE 2003-2013.

29.- Wallinga J, Teunis P, Kretzschmar M. Using social contact data to estimate age-specific transmission parameters for infectious respiratory spread agents. Am J Epidemiol 2006; 164: 936-44.

30.- Chowell G, Nishiura H, Bettencourt L M. Comparative estimation of the reproduction number for pandemic influenza from daily case notification data. J R Soc Interface 2007; 4: 155 66.

31.- Longini I M Jr., Halloran M E, Nizam A, Yang Y. Containing pandemic influenza with antiviral agents. Am J Epidemiol 2004; 159: 623-33.

32.- Ferguson N M, Cummings D A, Cauchemez S, Fraser C, Riley S, Meeyai A, et al. Strategies for containing an emerging influenza pandemic in Southeast Asia. Nature 2005; 437: 209-14.

33.- Centro de Documentación y Análisis Social de la Federación Venezolana de MaestrosCENDAS-FVM. [online]. Available at: [http:// www.cendasfvm.org/]. EL NACIONAL WEB. 18 DE NOVIEMBRE DE 2014 - 11:56 AM. [http://www.el-nacional.com/economia/Canastafamiliar-aumento-Bs-octubre_0_521947865. html].

34.- Gaceta Oficial $N^{\circ} 40.542 .17$ de noviembre de 2014. [online]. Disponible en: http://www. mp.gob.ve/c/document_library/get_file?p_1 id= $29950 \&$ folderId $=6047602 \&$ name $=$ DLFE- 8115 . pdf. (Accedido el 15 de agosto de 2014).

35.- OSELTAMIVIR (Tamifluß). [online]. Disponible en: [www.drougs.com/dosage/ tamiflu.html] (Accedido el 15 de agosto de 2014).

36.- Convenio Cambiario $N^{\circ} 31.02 / 12 / 2014$. [online]. Disponible en: [http://www.bcv.org. $\mathrm{ve} / \mathrm{c} 5 / \mathrm{sicad} 2 /$ sicad2-01.asp] (Accedido el $15 \mathrm{de}$ agosto de 2014).

37.- Welte R, Leidl R, Greiner W, Postma M, Krämer A, Kretzschmar M, et al. Health Economics of Infectious Diseases. In: Modern Infectious Disease Epidemiology: Concepts, Methods, Mathematical Models, and Public Health. 249-275. Springer New York, New York, NY, 2010.

38.- Centers for Disease Control and Prevention. Antiviral agents for the treatment and chemoprophylaxis of influenza Recommendations of the Advisory Committee on Immunization Practices (ACIP). MMWR Morb Mortal Wkly Rep 2011; 60 (1): 3-11.

39.- Sachs L. Angewandte Statistik, Anwendung statistischer Methoden. Springer-Verlag, Berlin, 1984. p. 262-4.

40.- Monto A S. Vaccines and antiviral drugs in pandemic preparedness. Emerg Infect Dis 2006; 12 (1): 55-60. doi:10.3201/eid1201.051068. 